
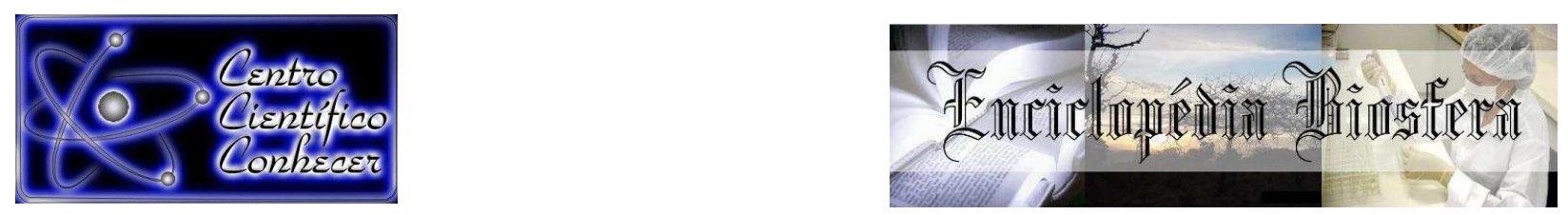

\title{
CYPERACEAE JUSS. E POACEAE BARN. DO HERBÁRIO PROFESSOR ALUÍZIO BITTENCOURT DA UNIVERSIDADE ESTADUAL DO MARANHÃO, CAXIAS, BRASIL
}

Paula Regina Pereira Martins ${ }^{1}$, Guilherme Sousa da Silva ${ }^{2}$, Gonçalo Mendes da Conceição ${ }^{3}$

1. Mestranda do Programa de Pós Graduação em Biodiversidade Ambiente e Saúde, Universidade Estadual do Maranhão, Caxias, MA (paulamartins_cx@hotmail.com)

2. Mestrando do Curso de Pós Graduação em Botânica do Instituto Nacional de

Pesquisas da Amazônia, Manaus, AM

3. Professor Doutor do Programa de Pós Graduação em Biodiversidade Ambiente e Saúde, Universidade Estadual do Maranhão, Centro de Estudos Superiores de Caxias

Recebido em: 08/04/2017 - Aprovado em: 10/06/2017 - Publicado em: 20/06/2017 DOI: 10.18677/EnciBio_2017A107

\begin{abstract}
RESUMO
A coleção do Herbário Professor Aluizio Bittencourt (HABIT), localizada na Universidade Estadual do Maranhão, campus Caxias/MA, é um elo importante para se estimar a flora do cerrado maranhense uma vez que nos últimos anos tem crescido as pesquisas botânicas da instituição de ensino superior em questão, promovendo a ampliação da coleção presente no acervo. Em virtude disso, este trabalho foi desenvolvido para verificar como as famílias de Cyperaceae Juss. e Poaceae Barn., pertencentes a ordem Poales, estão representadas no HABIT, uma vez que apresentam pouca representatividade nos herbários nacionais. Para se alcançar o objetivo da pesquisa, foi realizado um levantamento, através da contagem e revisão das espécies contidas no Herbário, separando e catalogando as mesmas a partir das informações contidas nas fichas de identificação de cada espécie, sendo obtido um total de 216 espécimes em 25 espécies distribuídas em oito gêneros de Cyperaceae Juss. Os gêneros Cyperus L. (08 spp.) e Rhynchospora Vahl (07 spp.) possuíram maior número de espécies. Enquanto a família Poaceae Barn. apresentou 78 espécimes em 34 espécies distribuídas em 19 gêneros, sendo o mais diversificado Paspalum L. (07 spp.). Apesar de poucos estudos realizados com as famílias em questão, grande parte das pesquisas registradas para o Estado, parte da iniciativa dos colaboradores do acervo.
\end{abstract}

PALAVRAS-CHAVE: Biodiversidade, coleção botânica, Poales 


\title{
REPRESENTATION OF CYPERACEAE JUSS. AND POACEAE BARN. IN THE HERBARY TEACHER ALUIIZIO BITTENCOURT OF THE STATE UNIVERSITY OF MARANHÃO, CAMPUS CAXIAS, MA
}

\begin{abstract}
The collection of the Teacher Aluizio Bittencourt Herbarium, located at the State University of Maranhão, on the campus of Caxias, MA, is an important link to estimate the bioflora of the Maranhão savannah since in recent years the botanical researches of the higher education institution in question, promoting the expansion of the collection present in the acquis. Because of that, this work was developed to verify how the families of Cyperaceae Juss. and Poaceae Barn., belonging to the order Poales, are represented in HABIT, since they presents little representation in the national herbariums. In order to reach the objective of the research, a survey was carried out, counting and reviewing the species, obtaining a total of 216 specimens in 25 species distributed in 8 genera of Cyperaceae Juss. The genera Cyperus L. (08 spp.) and Rynchospora Vahl (07 spp.) had a larger number of species. While, the Poaceae Barn. family presented 78 specimens with 34 species, distributed in 19 genera, being the most diversified Paspalum L. (07 spp.). Although few studies have been carried out with the families in question, most of the research carried out for the state, mainly Cyperaceae Juss., starts from the initiative of the collaborators of the collection.
\end{abstract}

KEYWORDS: Biodiversity, botanical collection, Poales

\section{INTRODUÇÃO}

Os herbários são centros que resguardam informações relevantes capazes de sustentar as estimativas da diversidade biológica de uma determinada região ou país, reúnem materiais vegetais, podendo ser todo o organismo ou parte deste, os quais são processados em etapas de prensagem para desidratação, limpeza, montagem em exsicatas e identificação. Além disso, PEIXOTO \& MORIM (2003) destacam algumas atribuições importantes de uma coleção botânica, tais como fornecer subsídios para o intercâmbio de informações e permuta de material biológico entre as comunidades científicas nacionais e internacionais, dar suporte para o desenvolvimento de pesquisa através de comparação de material, guardar exsicatas de trabalhos de pesquisas pregressas e disponibilizar material didático e científico para estudantes de graduação e de pós-graduação.

O Herbário Prof. Aluizio Bittencourt (HABIT), localizado no Centro de Estudos Superiores de Caxias/CESC, da Universidade Estadual do Maranhão/UEMA, representa a maior coleção do Maranhão, a segunda maior coleção da sub-região meio norte, (VIEIRA \& GASPER, 2015), além de possuir a maior coleção de briófitas do meio norte (CONCEIÇÃO, 2015), contando com aproximadamente 4.812 espécimes de angiospermas, distribuídas em 140 famílias, 73 espécies de briófitas em 20 famílias e 88 espécimes de Pteridófitas, em 14 famílias, caracterizando principalmente, o cerrado Maranhense (QUEIRÓS et al., 2014; CONCEIÇÃO, 2015).

O Cerrado é considerado uma das 34 áreas prioritárias para a conservação da biodiversidade (MITTERMEIER et al., 2005), apresentando elevado grau de endemismo em sua fauna e flora (MARINHO FILHO et al., 2010). As famílias Poaceae Barn. e Cyperaceae Juss., ambas pertencentes a ordem Poales (APG IV, 2016), apresentam destaque nesse bioma pela riqueza e distribuição das espécies (REZENDE et al., 2008; BOLDRINI \& LONGHI-WAGNER, 2011). Poales é a segunda maior ordem de monocotiledôneas, agrupa 16 famílias e cerca de 19000 
espécies herbáceas (APG III, 2009), com grande importância econômica e ecológica, principalmente nos ecossistemas campestres.

A família Poaceae Barn. inclui 793 gêneros e cerca de 11.000 espécies (OSBORNE et al., 2011; GPWG, 2013). No Brasil são indicados 225 gêneros e 1.486 espécies de Poaceae Barn. (FILGUEIRAS et al., 2015). Enquanto a família Cyperaceae Juss. possui amplitude cosmopolita, sendo a terceira maior família dentro das monocotiledôneas, possui cerca de 5.500 espécies descritas, distribuídas em 104 gêneros no mundo (GOVAERST et al., 2007) e no Brasil ocorrem aproximadamente 673 espécies, distribuídas em 39 gêneros (BFG, 2015). Apesar disso, com relação ao conhecimento da biodiversidade de Cyperaceae Juss., Alves et al., (2009) destacam a má representação da família nos herbários brasileiros, podendo ser resultado da falta de esforços aliada à ocorrência de muitas espécies raras ou de distribuição limitada o que pode ser um indicativo de uma extinção em potencial.

Em virtude da importância ecologia e econômica que Cyperaceae Juss. e Poaceae Barn. apresentam, atrelado ao pouco conhecimento de suas extensões territoriais, este estudo foi desenvolvido visando catalogar as espécies destas duas famílias pertencentes da ordem Poales que estão depositadas no HABIT para verificar a atual representação e contribuição destas para o Estado do Maranhão, tendo o cerrado como bioma em destaque.

\section{Local de estudo}

\section{MATERIAL E MÉTODOS}

A pesquisa foi realizada no Herbário Prof. Aluizio Bittencourt (HABIT), da Universidade Estadual do Maranhão, Campus Caxias/MA, que foi fundado em 1991. Em 2006 foi denominado Professor Aluizio Bittencourt, Farmacêutico-Bioquímico, Professor desde 1977 na Universidade Estadual do Maranhão, campus Caxias (QUEIRÓs et al., 2014; CONCEIÇÃO, 2015).

Atualmente a coleção botânica é composta por um crescente número de espécimes de vários grupos, Fanerógamas e Criptógamas, com destaque para a comunidade de Briófitas, representando principalmente a vegetação do cerrado maranhense, como resultado de pesquisas desenvolvidas por acadêmicos do Curso de Ciências Biológias Licenciatura e por mestrandos do Programa de PósGraduação em Biodiversidade, Ambiente e Saúde/PPGBAS da UEMA, campus Caxias/MA. Além disso, a coleção conta também com espécimes doados por Herbários de outros estados brasileiros. O mapa a seguir representa os municípios e localidades em que foram realizadas as coletas do material botânico em estudo que se encontra acervados no HABIT (Figura 1). 


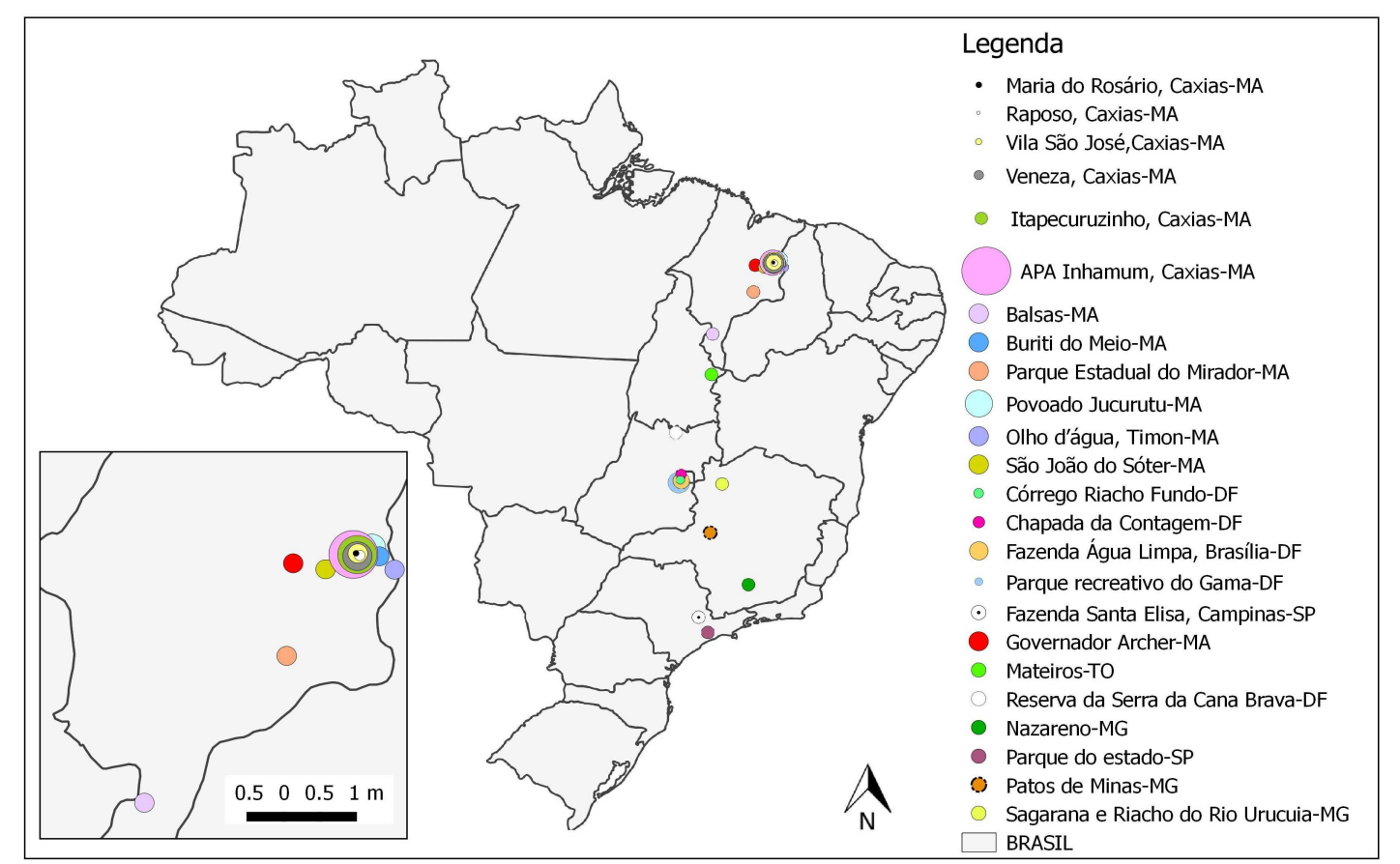

FIGURA 1. Municípios e localidades de coleta das Poales acervadas no Herbário Professor Aluízio Bittencourt, em destaque para o Estado do Maranhão. Fonte: OLíMPIO, A. P. M. (2017)

A preparação das exsicatas ocorre no interior do Laboratório de Botânica Vegetal (LABIVE), onde está localizada a coleção do HABIT, que se apresenta ordenada por famílias taxonômicas, depositadas em armários de aço sob refrigeração à $20^{\circ} \mathrm{C}$. Todo o material botânico encontra-se em processo de revisão taxonômica e as informações das etiquetas (nome científico, nome do coletor, local e data da coleta) estão sendo compiladas e adicionadas a um banco de dados digital, no programa "Faz Ficha", onde vem sendo confeccionadas etiquetas-padrão.

\section{Procedimentos metodológicos}

As análises das espécies de Cyperaceae Juss. e Poaceae Barn. foram realizadas com base no material herborizado e acervado no HABIT, onde se realizou a triagem dos espécimes pertencentes às duas famílias em questão, sendo estes depositados em armário separado para facilitar a análise. Após triagem, foi realizada limpeza, utilizando pincel de cerdas macias, para preservar a integridade do material.

Em seguida, alguns espécimes foram preparados em exsicatas, uma vez que havia uma considerável amostra embalada em jornais. Depois de todo material está preparado nas exsicatas devidamente etiquetadas, foram revisadas taxonomicamente, quando necessário, seguindo a lista de Espécies da Flora do Brasil 2020 em Construção (2016), literaturas especializadas (KEARNS et al., 1998; STRONG, 2006; TREVISAN \& BOLDRINI 2010; PRATA et al., 2013) e através de consultas a opera principia e typi digitalizados disponíveis online. Os dados obtidos foram compilados em tabela no Microsofit Excel 2010 para análise posterior.

\section{RESULTADOS E DISCUSSÃO}

No Herbário Prof. Aluizio Bittencourt, a família Cyperaceae Juss. está representada por um total de 216 espécimes, presentes em 25 espécies, estas distribuídas em 8 gêneros. O gênero representado por maior número de espécies 
presentes na coleção é o gênero Cyperus L. (08 spp.), seguido de Rhynchospora Vahl (07 spp.) (quadro 1, figura 3 A, C, D e E). A maioria dos gêneros de Cyperaceae são monotípicos, sendo que mais de dois terços das espécies pertencem a somente seis gêneros: Carex L. (ca. 2000), Cyperus L. (550), Fimbristylis Vahl (300), Rhynchospora Vahl (250), Scleria Berg. (250) e Eleocharis R. Br. (200) (GOETGHEBEUR, 1998). A família Cyperaceae Juss. é encontrada em diferentes ecossistemas com destaque para as áreas de campos, bordas de matas, dunas litorâneas, margem de rios e lagos e ambientes perturbados (ALVES et al., 2009).

PRATA et al. (2013) apontam que a riqueza de espécies não é uniformemente distribuída nas regiões do país. O que pode ser resultado da escassez de estudos aliado ao esforço de coleta. No Nordeste do Brasil, os estudos com Cyperaceae são escassos, com destaque para os trabalhos de LUCEÑO \& ALVES (1997), FREITAS et al., (2011), MENDES et al., (2010) e RIBEIRO et al. (2014). E para o estado do Maranhão, tem-se as colaborações de NUNES et al., (2012) e SANTOS et al., (2014) que listaram as espécies de Cyperaceae ocorrentes no município de Caxias/MA totalizando 31 espécies em sete gêneros, trabalhos estes apenas de levantamentos.

QUADRO 1. Espécies da família Cyperaceae Juss. depositadas na coleção Botânica do Herbário Prof. Aluizio Bittencourt/HABIT

\begin{tabular}{|c|c|c|c|c|}
\hline Gênero/Espécie & $\begin{array}{c}\text { Domínios } \\
\text { fitogeográficos }\end{array}$ & $\begin{array}{c}\text { Distribuição } \\
\text { geográfica }\end{array}$ & $\begin{array}{c}\text { Local de coleta, } \\
\text { ano }\end{array}$ & $\begin{array}{c}\text { № de } \\
\text { espécimes }\end{array}$ \\
\hline \multicolumn{5}{|l|}{ Bulbostylis Kunth. } \\
\hline $\begin{array}{l}\text { B. capillaris (L.) } \\
\text { C.B.Clarke }\end{array}$ & $\begin{array}{l}\text { AM, CA, CE, } \\
\text { MA, PP, PA. }\end{array}$ & $\begin{array}{l}\text { AC, AM, AP, } \\
\text { PA, RO, RR, } \\
\text { TO, AL, BA, } \\
\text { CE, MA, PB, } \\
\text { PE, PI, RN, } \\
\text { SE, DF, GO, } \\
\text { MS, MT, ES, } \\
\text { MG, RJ, SP, } \\
\text { PN, RS, SC. }\end{array}$ & $\begin{array}{l}\text { APA Buriti do } \\
\text { meio, } 2013\end{array}$ & 15 \\
\hline $\begin{array}{c}\text { B. consaguinea } \\
\text { (Kunth) } \\
\text { C.B.Clarke }\end{array}$ & $\begin{array}{l}\text { AM, CA, CE, } \\
M A, P P, P A .\end{array}$ & $\begin{array}{l}\text { AM, RO, RR, } \\
\text { TO, BA, PB, } \\
\text { PE, PI, DF, } \\
\text { GO, MG, SP, } \\
\text { PN, RS, SC. }\end{array}$ & $\begin{array}{l}\text { APA Buriti do } \\
\text { meio, } 2013\end{array}$ & 06 \\
\hline
\end{tabular}

\section{Cyperus L.}

PN, RS, SC.

\begin{tabular}{|c|c|c|c|c|}
\hline \multirow{4}{*}{$\begin{array}{l}\text { C. aggregatus } \\
\text { (Willd.) Endl. }\end{array}$} & \multirow{4}{*}{$\begin{array}{l}\text { AM, CA, CE, } \\
\text { MA, PP, PA. }\end{array}$} & \multirow{4}{*}{$\begin{array}{l}\text { AC, AM, AP, } \\
\text { PA, RO, RR, } \\
\text { TO, AL, BA, } \\
\text { CE, MA, PB, } \\
\text { PE, PI, RN, } \\
\text { SE, DF, GO, } \\
\text { MS, MT, ES, } \\
\text { MG, RJ, SP, } \\
\text { PN, RS, SC. }\end{array}$} & $\begin{array}{c}\text { Parque. } \\
\text { Estadual do } \\
\text { Mirador, sd }{ }^{*}\end{array}$ & 10 \\
\hline & & & $\begin{array}{l}\text { BR } 316 \text { Estação } \\
\text { de tratamento, } \\
\text { Balneário Maria } \\
\text { do rosário, } 2006\end{array}$ & 01 \\
\hline & & & $\begin{array}{c}\text { APA Inhamum, } \\
\text { MA, } 2013\end{array}$ & 15 \\
\hline & & & $\begin{array}{c}\text { APA Buriti do } \\
\text { Meio, MA, } 2013\end{array}$ & 52 \\
\hline
\end{tabular}




\begin{tabular}{|c|c|c|c|c|}
\hline C. digitatus Roxb. & $\begin{array}{c}\text { AM, CA, CE, } \\
\text { MA. }\end{array}$ & $\begin{array}{l}\text { AM, PA, BA, } \\
\text { CE, MA, PB, } \\
\text { MT, MG. }\end{array}$ & $\begin{array}{c}\text { APA Buriti do } \\
\text { Meio, MA, } 2013\end{array}$ & 03 \\
\hline \multirow[t]{2}{*}{ C. distans L. } & \multirow[t]{2}{*}{$\begin{array}{l}\text { AM, CA, CE, } \\
M A, P P, P A .\end{array}$} & \multirow{2}{*}{$\begin{array}{l}\text { AC, AP, PA, } \\
\text { RO, RR, TO, } \\
\text { AL, BA, CE, } \\
\text { MA, PB, PE, } \\
\text { PI, RN, SE, } \\
\text { DF, GO, MS, } \\
\text { MT, MG, RJ, } \\
\text { SP, RS. }\end{array}$} & $\begin{array}{l}\text { Buriti do meio, } \\
\text { MA } 2013\end{array}$ & 01 \\
\hline & & & $\begin{array}{c}\text { APA Inhamum, } \\
\text { MA, } 2013\end{array}$ & 02 \\
\hline \multirow{6}{*}{ C. haspan L. } & \multirow{6}{*}{$\begin{array}{l}\text { AM, CA, CE, } \\
\text { MA, PP, PA. }\end{array}$} & \multirow{6}{*}{$\begin{array}{c}\text { AC, AM, AP, } \\
\text { PA, RO, RR, } \\
\text { TO, AL, BA, } \\
\text { CE, MA, PB, } \\
\text { PE, PI, RN, } \\
\text { SE, GO, MS, } \\
\text { ES, MG, RJ, } \\
\text { SP, PN, RS, } \\
\text { SC. }\end{array}$} & $\begin{array}{c}\text { APA Inhamum, } \\
\text { MA, } 2012\end{array}$ & 04 \\
\hline & & & $\begin{array}{c}\text { Margem do Rio } \\
\text { Bom Gosto, MA, } \\
\text { sd }^{*}\end{array}$ & 01 \\
\hline & & & $\begin{array}{c}\text { Balneário } \\
\text { Veneza, MA, sd }\end{array}$ & 01 \\
\hline & & & $\begin{array}{l}\text { Sentido Caxias } \\
\text { - São João do } \\
\text { Sóter, MA, } \\
\text { Margem } \\
\text { Esquerda } 2006\end{array}$ & 03 \\
\hline & & & $\begin{array}{c}\text { Pov. Jucurutu, } \\
\text { MA, } 2006\end{array}$ & 03 \\
\hline & & & $\begin{array}{c}\text { Itapecuruzinho, } \\
\text { Margem do Rio } \\
\text { Itapecuru, MA, } \\
2006\end{array}$ & 01 \\
\hline \multirow{4}{*}{ C. iria L. } & \multirow{4}{*}{$\begin{array}{l}\text { AM, CA, CE, } \\
M A, P A, P P\end{array}$} & \multirow{4}{*}{$\begin{array}{l}\text { AC, AM, AP, } \\
\text { PA, RO, RR, } \\
\text { TO, AL, BA, } \\
\text { CE, MA, PB, } \\
\text { PE, PI, RN, } \\
\text { SE, DF, GO, } \\
\text { MS, MT, ES, } \\
\text { MG, RJ, SP, } \\
\text { PN, RS, SC. }\end{array}$} & $\begin{array}{c}\text { APA Inhamum, } \\
\text { MA, } 2006\end{array}$ & 01 \\
\hline & & & $\begin{array}{c}\text { APA Buriti do } \\
\text { Meio, MA, } 2013\end{array}$ & 01 \\
\hline & & & $\begin{array}{c}\text { Município } \\
\text { Governador } \\
\text { Archer, MA, sd }\end{array}$ & 02 \\
\hline & & & $\begin{array}{c}\text { Olho d'água, } \\
\text { Timon, MA, } \\
2008\end{array}$ & 01 \\
\hline \multirow{4}{*}{$\begin{array}{l}\text { C. Iuzulae (L.) } \\
\text { Retz. }\end{array}$} & \multirow[t]{4}{*}{$\begin{array}{l}\text { AM, CA, CE, } \\
\text { MA, PA, PP }\end{array}$} & \multirow{4}{*}{$\begin{array}{l}\text { AC, AM, AP, } \\
\text { PA, RO, RR, } \\
\text { TO, AL, BA, } \\
\text { CE, MA, PB, } \\
\text { PE, PI, RN, } \\
\text { SE, DF, GO, } \\
\text { MS, MT, ES, } \\
\text { MG, RJ, SP, } \\
\text { PN, RS, SC. }\end{array}$} & $\begin{array}{l}\text { APA Buriti do } \\
\text { Meio, MA, } 2013\end{array}$ & 01 \\
\hline & & & $\begin{array}{c}\text { APA Inhamum, } \\
\text { MA, } 2013\end{array}$ & 13 \\
\hline & & & $\begin{array}{c}\text { Parque Estadual } \\
\text { do Mirador, MA, } \\
2007\end{array}$ & 09 \\
\hline & & & $\begin{array}{l}\text { Vila são José } \\
\text { (próximo ao } \\
\text { riacho), Caxias, }\end{array}$ & 01 \\
\hline
\end{tabular}




\begin{tabular}{|c|c|c|c|c|}
\hline & & & MA, 2006 & \\
\hline $\begin{array}{c}\text { C. sphacelatus } \\
\text { Rottb. }\end{array}$ & $\begin{array}{c}\text { AM, CA, CE, } \\
\text { MA. }\end{array}$ & $\begin{array}{l}\text { AC, AM, AP, } \\
\text { PA, RO, RR, } \\
\text { TO, AL, BA, } \\
\text { CE, MA, PB, } \\
\text { PE, PI, RN, } \\
\text { SE, DF, GO, } \\
\text { MS, MT, ES, } \\
\text { MG, RJ, SP, } \\
\text { PN, RS, SC. }\end{array}$ & $\begin{array}{c}\text { APA Inhamum, } \\
\text { MA, } 2012\end{array}$ & 07 \\
\hline \multirow{4}{*}{$\begin{array}{c}\text { C. surinamensis } \\
\text { Rottb. }\end{array}$} & \multirow{4}{*}{$\begin{array}{l}\text { AM, CA, CE, } \\
\text { MA, PA, PP. }\end{array}$} & \multirow{4}{*}{$\begin{array}{l}\text { AC, AM, AP, } \\
\text { PA, RO, RR, } \\
\text { TO, AL, BA, } \\
\text { CE, MA, PB, } \\
\text { PE, PI, RN, } \\
\text { SE, DF, GO, } \\
\text { MS, MT, ES, } \\
\text { MG, RJ, SP, } \\
\text { PN, RS, SC. }\end{array}$} & $\begin{array}{c}\text { APA, Buriti do } \\
\text { meio, MA, } 2013\end{array}$ & 01 \\
\hline & & & $\begin{array}{c}\text { APA Inhamum, } \\
\text { MA, } 2013\end{array}$ & 09 \\
\hline & & & $\begin{array}{c}\text { Parque Estadual } \\
\text { do Mirador, MA, } \\
\text { sd }^{*}\end{array}$ & 01 \\
\hline & & & $\begin{array}{c}\text { Povoado } \\
\text { jacurutu, MA, } \\
2006\end{array}$ & 01 \\
\hline
\end{tabular}

\section{Eleocharis R.Br.}

\section{Fimbristylis Vahl}

E. geniculata (L.)

AM, CA, CE,

MA, PA, PP

PA, RR, TO,

AL, BA, CE,

MA, PB, PE,

PI, RN, SE,

DF, GO, MS,

Povoado

MT, ES, MG,

RJ, SP, PN,

Jacurutu, MA,

01

RS, SC.

\begin{tabular}{|c|c|c|c|c|}
\hline \multirow[t]{2}{*}{$\begin{array}{c}\text { F. miliacea (L.) } \\
\text { Vahl }\end{array}$} & \multirow[t]{2}{*}{$\begin{array}{l}\text { AM, CA, CE, } \\
\text { MA, PA, PP. }\end{array}$} & \multirow{2}{*}{$\begin{array}{l}\text { AC, AM, AP, } \\
\text { PA, RO, RR, } \\
\text { TO, AL, BA, } \\
\text { CE, MA, PB, } \\
\text { PE, PI, RN, } \\
\text { SE, DF, GO, } \\
\text { MS, MT, ES, } \\
\text { MG, RJ, SP, } \\
\text { PN, RS, SC. }\end{array}$} & $\begin{array}{l}\text { APA Buriti do } \\
\text { Meio, MA, } 2013\end{array}$ & 03 \\
\hline & & & $\begin{array}{c}\text { Povoado } \\
\text { jacurutu, MA, } \\
2006\end{array}$ & 01 \\
\hline
\end{tabular}

Fuirena Rottb.

AC, AM, AP,

APA Buriti do

03

Meio, MA, 2013

APA Inhamum,

MA, 2012

Povoado São

João do sóter

PA, RO, RR,

sentido Caxias, 


\begin{tabular}{|c|c|c|c|c|}
\hline \multirow{4}{*}{$\begin{array}{l}\text { F. umbellata } \\
\text { Rottb. }\end{array}$} & \multirow{4}{*}{$\begin{array}{l}\text { AM, CA, CE, } \\
\text { MA, PA, PP. }\end{array}$} & \multirow{4}{*}{$\begin{array}{l}\text { TO, AL, BA, } \\
\text { CE, MA, PB, } \\
\text { PE, PI, RN, } \\
\text { SE, DF, GO, } \\
\text { MS, MT, ES, } \\
\text { MG, RJ, SP, } \\
\text { PN, RS, SC. }\end{array}$} & $\begin{array}{c}\text { MA, margem } \\
\text { esquerda, } 2006\end{array}$ & \\
\hline & & & $\begin{array}{c}\text { Povoado } \\
\text { Jacurutu, MA, } \\
2006\end{array}$ & 01 \\
\hline & & & $\begin{array}{c}\text { Itapecuruzinho, } \\
\text { margem do Rio } \\
\text { Itapecuru, MA, } \\
2006\end{array}$ & 01 \\
\hline & & & $\begin{array}{c}\text { BR 316, estação } \\
\text { de tratamento } \\
\text { Balneário Maria } \\
\text { do rosário, MA, } \\
2006\end{array}$ & 01 \\
\hline \multicolumn{5}{|l|}{ Kyllinga Rottb. } \\
\hline \multirow[t]{2}{*}{$\begin{array}{l}\text { K. brevifolia } \\
\text { Rottb. }\end{array}$} & \multirow[t]{2}{*}{$\begin{array}{l}\text { AM, CA, CE, } \\
\text { MA, PA, PP. }\end{array}$} & \multirow{2}{*}{$\begin{array}{l}\text { AC, AM, AP, } \\
\text { PA, RO, RR, } \\
\text { TO, AL, BA, } \\
\text { CE, MA, PB, } \\
\text { PE, PI, RN, } \\
\text { SE, DF, GO, } \\
\text { MS, MT, ES, } \\
\text { MG, RJ, SP, } \\
\text { PN, RS, SC. }\end{array}$} & $\begin{array}{c}\text { Parque Estadual } \\
\text { do Mirador, MA, } \\
\text { sd }^{*}\end{array}$ & 03 \\
\hline & & & $\begin{array}{c}\text { APA Inhamum, } \\
\text { MA, } 2012\end{array}$ & 01 \\
\hline \multirow{4}{*}{ K. odorata Vahl } & \multirow{4}{*}{$\begin{array}{l}\text { AM, CA, CE, } \\
\text { MA, PA, PP. }\end{array}$} & \multirow{4}{*}{$\begin{array}{c}\text { AC, AM, AP, } \\
\text { PA, RO, RR, } \\
\text { TO, AL, BA, } \\
\text { CE, MA, PB, } \\
\text { PB, PI, RN, } \\
\text { SE, DF, GO, } \\
\text { MS, MT, } \\
\text { ES,MG, RJ, } \\
\text { SP, RS. }\end{array}$} & $\begin{array}{c}\text { APA Inhamum, } \\
\text { MA, } 2012\end{array}$ & 01 \\
\hline & & & $\begin{array}{c}\text { Itapecuruzinho, } \\
\text { margem do Rio } \\
\text { Itapecuru, MA, } \\
2006\end{array}$ & 01 \\
\hline & & & $\begin{array}{c}\text { Vila são José, } \\
\text { MA, } 2006\end{array}$ & 01 \\
\hline & & & $\begin{array}{c}\text { Balneário } \\
\text { Veneza, MA, sd* }\end{array}$ & 01 \\
\hline \multirow[t]{2}{*}{ K. vaginata Lam. } & \multirow[t]{2}{*}{$\begin{array}{l}\text { AM, CA, CE, } \\
\text { MA, PA, PP. }\end{array}$} & \multirow{2}{*}{$\begin{array}{l}\text { AC, AM, AP, } \\
\text { PA, RO, RR, } \\
\text { TO, AL, BA, } \\
\text { CE, MA, PB, } \\
\text { PE, PI, RN, } \\
\text { SE, DF, GO, } \\
\text { MS, MT, ES, } \\
\text { MG, RJ, SP, } \\
\text { PN, RS, SC. }\end{array}$} & $\begin{array}{l}\text { APA Buriti do } \\
\text { Meio, MA, } 2013\end{array}$ & 01 \\
\hline & & & $\begin{array}{c}\text { Margem do Rio } \\
\text { Bom Gosto, MA, } \\
\text { sd }^{*}\end{array}$ & 01 \\
\hline \multicolumn{5}{|c|}{ Rhynchospora Vahl } \\
\hline $\begin{array}{l}\text { R. cephalotes (L.) } \\
\text { Vahl }\end{array}$ & $\begin{array}{c}\text { AM, CA, CE, } \\
\text { MA. }\end{array}$ & $\begin{array}{l}\mathrm{AC}, \mathrm{AM}, \mathrm{AP}, \\
\mathrm{PA}, \mathrm{RO}, \mathrm{RR}, \\
\mathrm{TO}, \mathrm{AL}, \mathrm{BA},\end{array}$ & n.i. ${ }^{\star *}, 2008$ & 02 \\
\hline
\end{tabular}




\begin{tabular}{|c|c|c|c|c|}
\hline & & $\begin{array}{l}\text { CE, MA, PB, } \\
\text { PE, PI, RN, } \\
\text { SE, DF, GO, } \\
\text { MS, MT, MG. }\end{array}$ & $\begin{array}{c}\text { Povoado } \\
\text { Jacurutu, MA, } \\
2006\end{array}$ & 01 \\
\hline $\begin{array}{l}\text { R. consaguinea } \\
\text { (Kunth) Boeckel }\end{array}$ & $\mathrm{AM}, \mathrm{CA}, \mathrm{CE}$ & $\begin{array}{l}\text { TO, BA, MA, } \\
\text { PI, DF, GO, } \\
\text { MS, MT, MG, } \\
\text { SP, PN. }\end{array}$ & $\begin{array}{l}\text { Fazenda Água } \\
\text { Limpa- univ. de } \\
\text { Brasília, DF } \\
\text { (Cerrado de } \\
\text { altitude.), } 2003\end{array}$ & 01 \\
\hline $\begin{array}{l}\text { R. corymbosa } \\
\text { (L.) Britton, }\end{array}$ & $\begin{array}{l}\text { AM, CA, CE, } \\
\text { MA, PA, PP. }\end{array}$ & $\begin{array}{l}\text { AC, AM, AP, } \\
\text { PA, RO, RR, } \\
\text { TO, AL, BA, } \\
\text { CE, MA, PB, } \\
\text { PE, PI, RN, } \\
\text { SE, DF, GO, } \\
\text { MS, MT, ES, } \\
\text { MG, RJ, SP, } \\
\text { PN, RS, SC. }\end{array}$ & $\begin{array}{l}\text { APA Buriti do } \\
\text { meio, MA, } 2013\end{array}$ & 01 \\
\hline \multirow{4}{*}{ R. exaltata Kunth } & \multirow{4}{*}{$\begin{array}{c}\text { AM, CA, CE, } \\
\text { MA. }\end{array}$} & \multirow{4}{*}{$\begin{array}{l}\text { AC, AM, AP, } \\
\text { PA, RO, RR, } \\
\text { TO, AL, BA, } \\
\text { CE, MA, PB, } \\
\text { PE, PI, RN, } \\
\text { SE, DF, GO, } \\
\text { MS, MT,ES, } \\
\text { MG, RJ, SP, } \\
\text { PN, SC. }\end{array}$} & $\begin{array}{c}\text { APA, Buriti do } \\
\text { meio, MA, } 2013\end{array}$ & 06 \\
\hline & & & $\begin{array}{c}\text { Parque Estadual } \\
\text { do Mirador, MA, } \\
2007\end{array}$ & 02 \\
\hline & & & $\begin{array}{l}\text { Sentido Caxias } \\
\text { - São João do } \\
\text { Sóter, MA, } \\
\text { Margem Esq., } \\
2006\end{array}$ & 01 \\
\hline & & & $\begin{array}{c}\text { Balneário Maria } \\
\text { do Rosário, MA, } \\
2006\end{array}$ & 02 \\
\hline $\begin{array}{c}R . \\
\text { holoschoenoides } \\
\text { (Rich.) Herter }\end{array}$ & $\begin{array}{l}\text { AM, CA, CE, } \\
M A, P A, P P .\end{array}$ & $\begin{array}{l}\text { AC, AM, AP, } \\
\text { PA, RO, RR, } \\
\text { TO, AL, BA, } \\
\text { CE, MA, PB, } \\
\text { PE, PI, RN, } \\
\text { SE, DF, GO, } \\
\text { MS, MT, ES, } \\
\text { MG, RJ, SP, } \\
\text { PN, RS, SC. }\end{array}$ & $\begin{array}{c}\text { Margem do Rio } \\
\text { bom gosto, MA, } \\
\text { sd }^{*}\end{array}$ & 01 \\
\hline \multirow{3}{*}{$\begin{array}{l}\text { R. riparia (Nees) } \\
\text { Boeckeler }\end{array}$} & \multirow{3}{*}{$\begin{array}{l}\text { AM, CA, CE, } \\
M A, P A, P P .\end{array}$} & \multirow{3}{*}{$\begin{array}{l}\text { AC, AM, AP, } \\
\text { PA, RO, RR, } \\
\text { TO, AL, BA, } \\
\text { CE, MA, PB, } \\
\text { PE, PI, RN, } \\
\text { SE, DF, GO, } \\
\text { MS, MT, ES, } \\
\text { MG, RJ, SP, } \\
\text { PN, RS, SC. } \\
\text { AC, AM, PA, }\end{array}$} & $\begin{array}{l}\text { APA Buriti do } \\
\text { meio, MA, } 2013\end{array}$ & 03 \\
\hline & & & $\begin{array}{l}\text { APA Inhamum, } \\
\text { MA, } 2012\end{array}$ & 01 \\
\hline & & & & \\
\hline
\end{tabular}




\begin{tabular}{|c|c|c|c|c|}
\hline R. tenuis Link & $\begin{array}{l}\text { AM, CA, CE, } \\
\text { MA, PA, PP. }\end{array}$ & $\begin{array}{c}\text { RO, RR, TO, } \\
\text { AL, BA, CE, } \\
\text { MA, PB, PE, } \\
\text { PI, RN, SE, } \\
\text { DF, GO, MS, } \\
\text { MT, ES, MG, } \\
\text { RJ, SP, PN, } \\
\text { RS, SC. }\end{array}$ & $\begin{array}{c}\text { Margem do Rio } \\
\text { Bom Gosto, MA, } \\
\text { sd }^{*}\end{array}$ & 01 \\
\hline \multicolumn{5}{|c|}{ Scleria P.J.Bergius } \\
\hline S. bracteata Cav. & $\begin{array}{c}\text { AM, CA, CE, } \\
\text { MA, PP. }\end{array}$ & $\begin{array}{l}\text { AM, AP, PA, } \\
\text { RO, TO, AL, } \\
\text { BA, CE, MA, } \\
\text { PB, PE, PI, } \\
\text { RN, SE, DF, } \\
\text { GO, MS, MT, } \\
\text { ES, MG, RJ, } \\
\text { SP. }\end{array}$ & $\begin{array}{c}\text { Município de } \\
\text { Patos de Minas, } \\
\text { MG, } 1950\end{array}$ & 01 \\
\hline $\begin{array}{l}\text { S. microcarpa } \\
\text { Nees ex Kunth }\end{array}$ & $\begin{array}{l}\text { AM, CA, CE, } \\
\text { MA, PA, PP. }\end{array}$ & $\begin{array}{l}\text { AC, AM, AP, } \\
\text { PA, RO, RR, } \\
\text { TO, AL, BA, } \\
\text { CE, MA, PB, } \\
\text { PE, PI, RN, } \\
\text { SE, DF, GO, } \\
\text { MS, MT, ES, } \\
\text { MG, RJ, SP, } \\
\text { PN, SC. }\end{array}$ & $\begin{array}{l}\text { APA Buriti do } \\
\text { meio, MA, sd }\end{array}$ & 02 \\
\hline $\begin{array}{l}\text { Total de } \\
\text { espécimes: }\end{array}$ & & & & 216 \\
\hline
\end{tabular}

Poaceae Bar. até o momento está representada na coleção do HABIT por 78 espécimes, em 34 espécies, distribuídas em 19 gêneros. A família se destaca como uma das mais ricas em ambientes savânicos, como o cerrado, campo rupestre e restingas (RUA, 2006). Os gêneros que se apresentam representados com maior número de espécies no HABIT foram Paspalum L. (08), seguido de Panicum L. (03), Axonopus P. Beauv. (03) e Schizachyrium Ness (03) (Quadro 2, Figura 3 G, I e L). Apesar disso, estes gêneros estão pouco representados na atual coleção, uma vez que apresentam-se bastante diversificados dentro da família, principalmente Paspalum, com cerca de 400 espécies nos trópicos e subtrópicos e aproximadamente 202 espécies no Brasil, apresenta forte potencial econômico nas atividades de forrageio em áreas de pastagens no país (VALLS \& OLIVEIRA, 2010). $\mathrm{E}$, Panicum que compreende cerca de 400 espécies em regiões pantropicais (ALISCIONI et al., 2003). A baixa representação de Poaceae no HABIT pode atribuir-se a ausência de profissionais específicos trabalhando com a família na instituição. 
QUADRO 2. Espécies da família Poaceae Barn. depositadas na coleção Botânica do Herbário Prof. Aluizio Bittencourt/HABIT

\begin{tabular}{|c|c|c|c|c|}
\hline Gênero/espécie & $\begin{array}{c}\text { Domínios } \\
\text { fitogeográficos }\end{array}$ & $\begin{array}{l}\text { Distribuição } \\
\text { geográfica }\end{array}$ & $\begin{array}{l}\text { Local de } \\
\text { coleta, ano }\end{array}$ & $\begin{array}{c}\text { N de } \\
\text { espécimes }\end{array}$ \\
\hline \multicolumn{5}{|l|}{ Andropogon L. } \\
\hline $\begin{array}{c}\text { A. angustatus (J. Presl) } \\
\text { Steud. }\end{array}$ & AM, CA, CE. & $\begin{array}{l}\text { RO, RR, CE, } \\
\text { MA, PB, PI, } \\
\text { RN, GO,MT. }\end{array}$ & $\begin{array}{c}\text { APA } \\
\text { Inhamum, } \\
\text { MA, 2010 }\end{array}$ & 01 \\
\hline \multirow[t]{2}{*}{ A. selloanus Hack } & \multirow[t]{2}{*}{$\begin{array}{l}\text { AM, CA, CE } \\
\text { MA, PP. }\end{array}$} & \multirow{2}{*}{$\begin{array}{l}\text { AM, PA, RR, } \\
\text { TO, BA, CE, } \\
\text { MA, PB, PE, } \\
\text { PI, RN, SE, } \\
\text { DF, GO, MS, } \\
\text { MG, ES, } \\
\text { MG, RJ, SP, } \\
\text { PN, RS, SC. }\end{array}$} & $\begin{array}{c}\text { Município } \\
\text { Nazareno, } \\
\text { MG, km 28, } \\
\text { rodovia } \\
\text { sentido São } \\
\text { João Del rei, } \\
1987\end{array}$ & 02 \\
\hline & & & $\begin{array}{c}\text { Entre } \\
\text { Sagarana e } \\
\text { Riacho do } \\
\text { Rio Urucuia, } \\
\text { MG, } 2000\end{array}$ & 01 \\
\hline \multicolumn{5}{|l|}{ Aristida L. } \\
\hline \multirow[b]{2}{*}{ A. longifolia Trin. } & \multirow[b]{2}{*}{ AM, CA, CE. } & \multirow{2}{*}{$\begin{array}{c}\text { AM, AP,PA, } \\
\text { RR, TO, AL, } \\
\text { BA, CE, MA, } \\
\text { PB, PE, PI, } \\
\text { RN, SE, DF, } \\
\text { GO, MS, } \\
\text { MT, MG. }\end{array}$} & $\begin{array}{c}\text { APA } \\
\text { Inhamum, } \\
\text { MA, 2010 }\end{array}$ & 02 \\
\hline & & & $\begin{array}{c}\text { Margem do } \\
\text { Rio Tem } \\
\text { Medo, } \\
\text { Balsas, MA, } \\
1997\end{array}$ & 01 \\
\hline \multicolumn{5}{|l|}{ Axonopus P. Beauv. } \\
\hline $\begin{array}{c}\text { A. eminens (Neis) G.A. } \\
\text { Black }\end{array}$ & $\mathrm{AM}, \mathrm{CA}, \mathrm{CE}$. & $\begin{array}{c}\text { AM, BA, MA, } \\
\text { SE, DF, GO, } \\
\text { MT, MG. }\end{array}$ & $\begin{array}{c}\text { Balsas, MA, } \\
1997\end{array}$ & 03 \\
\hline $\begin{array}{l}\text { A. marginatus (Trin.) } \\
\text { Chase }\end{array}$ & $\begin{array}{l}\text { AM, CA, CE, } \\
\text { MA. }\end{array}$ & $\begin{array}{c}\text { AM, PA, TO, } \\
\text { AL, BA, CE, } \\
\text { MA, PB, PE, } \\
\text { PI, RN, SE, } \\
\text { DF, GO, MS, } \\
\text { MT, ES, MG, } \\
\text { RJ, SP, PN, } \\
\text { SC. }\end{array}$ & $\begin{array}{l}\text { Balsas, MA, } \\
1996\end{array}$ & 01 \\
\hline $\begin{array}{c}\text { A. polydactylus (Steud.) } \\
\text { Dedecca }\end{array}$ & $\begin{array}{l}\text { AM, CA, CE, } \\
\text { SC. }\end{array}$ & $\begin{array}{c}\text { AM, PA, TO, } \\
\text { AL, BA, CE, } \\
\text { MA, PB, PE, } \\
\text { PI, RN, SE, } \\
\text { GO, MT, } \\
\text { MG, SP, PN. }\end{array}$ & $\begin{array}{c}\text { APA } \\
\text { Inhamum, } \\
\text { MA, } 2010\end{array}$ & 02 \\
\hline \multicolumn{5}{|l|}{ Bambusa Schreb. } \\
\hline & & & Comunidade & \\
\hline
\end{tabular}




\begin{tabular}{|c|c|c|c|c|}
\hline $\begin{array}{l}\text { B. vulgaris Schrad. Ex } \\
\text { J.C. Wendl. }\end{array}$ & $\begin{array}{c}\text { AM, CA, CE, } \\
\text { MA, PA. }\end{array}$ & $\begin{array}{l}\text { AL, BA, PE, } \\
\text { DF, GO, MS, } \\
\text { MT, MG, RJ, } \\
\text { SP, PN, SC. }\end{array}$ & $\begin{array}{c}\text { quilombola o } \\
\text { Olho d'água } \\
\text { Raposo, } \\
\text { Caxias, MA, } \\
2009\end{array}$ & 05 \\
\hline \multicolumn{5}{|l|}{ Chaetium Nees } \\
\hline C. festucoides Nees & CA, MA. & $\begin{array}{l}\mathrm{CE}, \mathrm{MA}, \mathrm{PB}, \\
\text { SE. }\end{array}$ & $\begin{array}{c}\text { APA } \\
\text { Inhamum, } \\
\text { MA, 2010 }\end{array}$ & 02 \\
\hline \multicolumn{5}{|l|}{ Ctenium Panz. } \\
\hline $\begin{array}{l}\text { C. chapadense (Trin.) } \\
\text { Döll }\end{array}$ & CA, CE. & $\begin{array}{l}\text { BA, DF, GO, } \\
\text { MT, MG. }\end{array}$ & $\begin{array}{l}\text { Parque } \\
\text { recreativo } \\
\text { do Gama, } \\
\text { DF, } 2000\end{array}$ & 01 \\
\hline \multicolumn{5}{|l|}{ Echinolaena Desv. } \\
\hline E. inflexa (Poir) Chase & $\begin{array}{l}\text { AM, CA, CE } \\
\text { MA. }\end{array}$ & $\begin{array}{l}\text { AC, AM, AP, } \\
\text { PA, RO, RR, } \\
\text { TO, BA, CE, } \\
\text { MA, PB, PE, } \\
\text { PI, SE, DF, } \\
\text { GO, MS, } \\
\text { MT, ES, MG, } \\
\text { RJ, SP, PN. }\end{array}$ & $\begin{array}{c}\text { Chapada da } \\
\text { Contagem, } \\
35 \mathrm{~km} \text { por } \\
\text { estrada do } \\
\text { Balão, DF, } \\
1980\end{array}$ & 02 \\
\hline \multicolumn{5}{|c|}{ Hyparrhenia Ander. ex E. Fourn. } \\
\hline H. rufa (Ness) Stapf. & $\begin{array}{l}\text { AM, CA, CE } \\
\text { MA, PA. }\end{array}$ & $\begin{array}{c}\text { AM, PA, } \\
\text { RO, RR, TO, } \\
\text { BA, CE, MA, } \\
\text { PB, PE, PI, } \\
\text { RN, DF, GO, } \\
\text { MS, MT, ES, } \\
\text { MG, RJ, SP, } \\
\text { PN, RS, SC. }\end{array}$ & $\begin{array}{c}\text { Município de } \\
\text { Minaçu, } \\
\text { Reserva da } \\
\text { Serra da } \\
\text { Cana Brava, } \\
\text { centro } \\
\text { oeste, DF, } \\
1995 \\
\end{array}$ & 03 \\
\hline \multicolumn{5}{|l|}{ Melinis P. Beauv. } \\
\hline $\begin{array}{c}\text { M. minutiflora } P \text {. } \\
\text { Beauv. }\end{array}$ & $\begin{array}{c}\text { AM, CA, CE } \\
\text { MA. }\end{array}$ & $\begin{array}{c}\text { AM, AP, PA, } \\
\text { RO, TO, BA, } \\
\text { CE, PB, PE, } \\
\text { DF, GO, } \\
\text { MS,MT, ES, } \\
\text { MG, RJ, SP, } \\
\text { PN, RS, SC. }\end{array}$ & $\begin{array}{c}\text { Córrego } \\
\text { Riacho } \\
\text { Fundo, } \\
\text { próximo ao } \\
\text { aeroporto, } \\
\text { DF, } 1980\end{array}$ & 01 \\
\hline \multicolumn{5}{|c|}{ Megathyrsus (Pilg.) B.K. Simon \& S.W.L. Jacobs } \\
\hline $\begin{array}{l}\text { M. maximus (Jacq.) } \\
\text { B.K. Simon \& S.W.L. } \\
\text { Jacobs }\end{array}$ & $\begin{array}{l}\text { AM, CA, CE } \\
\text { MA, PA. }\end{array}$ & $\begin{array}{c}\text { AM, BA, CE, } \\
\text { PE, RN, DF, } \\
\text { GO, MS, } \\
\text { MG, MG, } \\
\text { RJ, SP, PN. }\end{array}$ & $\begin{array}{c}\text { APA } \\
\text { Inhamum, } \\
\text { MA, } 2010\end{array}$ & 02 \\
\hline \multicolumn{5}{|l|}{ Merostachys Spreng. } \\
\hline & & & $\begin{array}{l}\text { Parque do } \\
\text { estado, Sub- }\end{array}$ & \\
\hline
\end{tabular}




\begin{tabular}{c|c|c|c|c}
\hline $\begin{array}{c}\text { M. plurifora Munro ex } \\
\text { E.G.Camus }\end{array}$ & MA. & RJ, SP, SC. & $\begin{array}{c}\text { bosque da } \\
\text { mata em } \\
\text { estudo, SP, } \\
1958\end{array}$ & 01 \\
\hline M. speciosa Spreng. & MA. & $\begin{array}{c}\text { MG, SP, RS, } \\
\text { SC. }\end{array}$ & $\begin{array}{c}\text { Fazenda } \\
\text { Santa Elisa, } \\
\text { Campinas, } \\
\text { SP, 1962 }\end{array}$ & 02 \\
\hline
\end{tabular}

\section{Panicum L.}

\begin{tabular}{|c|c|c|c|c|}
\hline P. cervicatum Chase & $\begin{array}{c}\text { AM, CA, CE } \\
\text { MA, PA. }\end{array}$ & $\begin{array}{l}\text { PA, RO, BA, } \\
\text { MA, PB, DF, } \\
\text { GO, MS, } \\
\text { MT, ES, MG, } \\
\text { SP, SC. }\end{array}$ & $\begin{array}{c}\text { Balsas, MA, } \\
1997\end{array}$ & 01 \\
\hline $\begin{array}{l}\text { P. ligulare Nees ex } \\
\text { Trin. }\end{array}$ & $\begin{array}{c}\text { AM, CA, CE } \\
\text { MA, PA. }\end{array}$ & $\begin{array}{c}\text { AM, PA, TO, } \\
\text { BA, MA,DF, } \\
\text { GO, MS, } \\
\text { MT, MG. }\end{array}$ & $\begin{array}{l}\text { Balsas, } \\
\text { MA,1997 }\end{array}$ & 02 \\
\hline P. millegrana Poir. & $\begin{array}{l}\text { AM, CA, CE, } \\
\text { MA, PA, PP. }\end{array}$ & $\begin{array}{l}\text { AC, AM, PA, } \\
\text { RO, RR, TO, } \\
\text { AL, BA, CE, } \\
\text { MA, PB, PE, } \\
\text { PI, RN, SE, } \\
\text { DF, GO, MS, } \\
\text { MT, ES, MG, } \\
\text { RJ, SP, PN, } \\
\text { RS, SC. }\end{array}$ & $\begin{array}{l}\text { Parque } \\
\text { recreativo } \\
\text { do Gama, } \\
\text { Centro- } \\
\text { oeste, DF, } \\
2000\end{array}$ & 01 \\
\hline \multicolumn{5}{|l|}{ Paspalum L. } \\
\hline P. ellipticum Döll. & $\begin{array}{l}\text { CE, MA, PA, } \\
\text { PP. }\end{array}$ & $\begin{array}{c}\text { BA, MA, DF, } \\
\text { GO, MS, } \\
\text { MT, MG, SP, } \\
\text { PN, RS, SC. }\end{array}$ & $\begin{array}{l}\text { Balsas, } \\
\text { MA,1997 }\end{array}$ & 01 \\
\hline $\begin{array}{l}P . \text { eucomum Nees ex } \\
\text { Trin. }\end{array}$ & CE. & $\begin{array}{l}\text { DF, GO, MT, } \\
\text { MG, SP, PN. }\end{array}$ & $\begin{array}{l}\text { Município de } \\
\text { Mateiros, } \\
\text { TO, } 2001 \\
\end{array}$ & 02 \\
\hline P. foliiforme S.Denham & $\begin{array}{l}\text { AM, CE, MA, } \\
\text { PA. }\end{array}$ & $\begin{array}{l}\text { AC, PA, RR, } \\
\text { TO, BA, MA, } \\
\text { DF, GO, MS, } \\
\text { MT, MG, SP. }\end{array}$ & $\begin{array}{l}\text { Balsas, } \\
\text { MA,1997 }\end{array}$ & 01 \\
\hline \multirow[t]{2}{*}{$P$. gardenerianum Nees } & \multirow[t]{2}{*}{$\mathrm{AM}, \mathrm{CA}, \mathrm{CE}$} & \multirow{2}{*}{$\begin{array}{l}\text { AM, AP, PA, } \\
\text { RR, TO, BA, } \\
\text { CE, MA, PB, } \\
\text { PE, PI, RN, } \\
\text { SE, DF, GO, } \\
\text { MS, MT. }\end{array}$} & $\begin{array}{c}\text { Balsas, MA, } \\
1997\end{array}$ & 01 \\
\hline & & & $\begin{array}{c}\text { APA } \\
\text { Inhamum, } \\
\text { MA, 2010 }\end{array}$ & 02 \\
\hline \multirow[t]{2}{*}{$P$. guttatum Trin. } & CE, MA. & $\begin{array}{l}\text { DF, GO, MS, } \\
\text { MT, MG, SP, } \\
\text { PN. }\end{array}$ & $\begin{array}{c}\text { Balsas, } \\
\text { MA,1996 }\end{array}$ & 02 \\
\hline & & & Margens do & \\
\hline
\end{tabular}




\begin{tabular}{|c|c|c|c|c|}
\hline$P$. lanciflorum Trin. & AM, CE. & $\begin{array}{l}\text { PA, RO, TO, } \\
\text { BA, MA, DF, } \\
\text { GO, MT. }\end{array}$ & $\begin{array}{c}\text { Rio Tem } \\
\text { Medo, } \\
\text { saindo da } \\
\text { vila, sd } \\
\text { Balsas, MA, } \\
1997 \\
\end{array}$ & 04 \\
\hline$P$. lineare Trin. & $\begin{array}{c}\text { CA, CE, MA, } \\
\text { PA. }\end{array}$ & $\begin{array}{l}\text { TO, BA, DF, } \\
\text { GO, MS, } \\
\text { MT, MG, SP, } \\
\text { PN. }\end{array}$ & $\begin{array}{l}\text { Balsas, } \\
\text { MA, } 1997\end{array}$ & 01 \\
\hline P. multicaule Poir. & $\begin{array}{c}\text { AM, CA, CE, } \\
\text { MA, PA. }\end{array}$ & $\begin{array}{l}\text { AM, AM, PA, } \\
\text { RO, RR, TO, } \\
\text { BA, MA, PB, } \\
\text { PE, PI, RN, } \\
\text { DF, GO, MS, } \\
\text { MT, ES, MG, } \\
\text { SP. }\end{array}$ & $\begin{array}{c}\text { Balsas, MA, } \\
1997\end{array}$ & 04 \\
\hline \multicolumn{5}{|l|}{ Raddiella Swallen } \\
\hline $\begin{array}{l}\text { R. esenbeckii (Steud.) } \\
\text { C.E.Calderón \& } \\
\text { Soderstr. }\end{array}$ & $\begin{array}{c}\text { AM, CA, CE, } \\
\text { MA. }\end{array}$ & $\begin{array}{l}\text { AM, AP, PA, } \\
\text { RO, RR, TO, } \\
\text { BA, MA, PI, } \\
\text { DF, GO, MS, } \\
\text { MT, MG, SP, } \\
\text { PN. }\end{array}$ & $\begin{array}{c}\text { Balsas, } \\
\text { morro antes } \\
\text { do Rio Tem } \\
\text { Medo, MA, } \\
1997\end{array}$ & 01 \\
\hline \multicolumn{5}{|l|}{ Rugoloa Zuloaga } \\
\hline R. pilosa & AM, MA. & $\begin{array}{l}\text { AC, AM, AP, } \\
\text { PA, RO, RR, } \\
\text { TO, BA, CE, } \\
\text { MA, PB, PE, } \\
\text { PI, DF, GO, } \\
\text { MS, MT, ES, } \\
\text { MG, RJ, SP, } \\
\text { PN, RS, SC. }\end{array}$ & $\begin{array}{c}\text { APA } \\
\text { Inhamum, } \\
\text { MA, 2010 }\end{array}$ & 02 \\
\hline \multicolumn{5}{|l|}{ Sacciolepis Nash } \\
\hline $\begin{array}{c}\text { S. angustissima. } \\
\text { (Hochst. ex Steud.) } \\
\text { Kuhlm. }\end{array}$ & AM, CE. & $\begin{array}{l}\text { AM, RO, BA, } \\
\text { GO, MS, } \\
\text { MT, MG, } \\
\text { PN, RS. }\end{array}$ & $\begin{array}{c}\text { APA } \\
\text { Inhamum, } \\
\text { MA, 2010 }\end{array}$ & 01 \\
\hline \multicolumn{5}{|l|}{ Schizachyrium Nees } \\
\hline $\begin{array}{c}\text { S. microstachyum } \\
\text { (Desv. ex Ham.) } \\
\text { Roseng., B.R. Arrill. \& } \\
\text { Izag. }\end{array}$ & $\begin{array}{l}\text { AM, CE, MA, } \\
\text { PP. }\end{array}$ & $\begin{array}{l}\text { AM, BA, MA, } \\
\text { PE, DF, GO, } \\
\text { MS, MT, } \\
\text { MG, RJ, SP, } \\
\text { PN, RS, SC. }\end{array}$ & $\begin{array}{c}\text { Balsas, MA, } \\
1997\end{array}$ & 02 \\
\hline $\begin{array}{l}\text { S. sanguineum } \\
\text { (Retz.) Alston }\end{array}$ & $\begin{array}{c}\text { AM, CA, CE, } \\
\text { MA, PP. }\end{array}$ & $\begin{array}{l}\text { AM, AP, PA, } \\
\text { RO, RR, TO, } \\
\text { BA, MA, PB, } \\
\text { DF, GO, MS, } \\
\text { MT, ES, MG, } \\
\text { RJ, SP, PN, }\end{array}$ & $\begin{array}{c}\text { Balsas, MA, } \\
1997\end{array}$ & 02 \\
\hline
\end{tabular}




\begin{tabular}{c|c|c|c|c}
\hline & & RS. & & \\
\hline & & AC, AM, & & \\
& & AP,PA, RO, & \\
RR, TO, AL, & Balsas, & \\
S. scoparium (Michx.) & AM, CA, CE, & BA, CE, MA, & prox. ao Rio & 02 \\
& MA, PP. & PE, DF, GO, & Tem Medo, & \\
& & MS, MT, & MA, 1997 & \\
& & MG, RJ, SP, & & \\
& & PN, RS, SC. & & \\
\hline
\end{tabular}

Streptostachys Desv.

\begin{tabular}{|c|c|c|c|c|}
\hline \multirow[b]{2}{*}{ S. asperifolia Desv. } & \multirow[b]{2}{*}{$\begin{array}{c}\text { AM, CA, CE, } \\
\text { MA. }\end{array}$} & \multirow{2}{*}{$\begin{array}{l}\text { AM, PA, RR, } \\
\text { TO, AL, BA, } \\
\text { CE, MA, PB, } \\
\text { PE, PI, RN, } \\
\text { SE, ES. }\end{array}$} & $\begin{array}{c}\text { Balsas, MA, } \\
1997\end{array}$ & 01 \\
\hline & & & $\begin{array}{c}\text { APA } \\
\text { Inhamum, } \\
\text { MA, 2010 }\end{array}$ & 09 \\
\hline \multicolumn{5}{|l|}{ Trachypogon Nees } \\
\hline \multirow[b]{2}{*}{ T. spicatus (L.f.) Kuntze } & \multirow[b]{2}{*}{$\begin{array}{c}\text { AM, CA, CE, } \\
\text { MA. }\end{array}$} & \multirow{3}{*}{$\begin{array}{l}\text { AM, AP, PA, } \\
\text { RO, RR, TO, } \\
\text { BA, MA, PB, } \\
\text { PI, RN, DF, } \\
\text { GO, MS, } \\
\text { MT, MG, SP. }\end{array}$} & $\begin{array}{c}\text { Balsas, } \\
\text { MA.1997 }\end{array}$ & 01 \\
\hline & & & $\begin{array}{c}\text { APA } \\
\text { Inhamum, } \\
\text { MA, } 2010\end{array}$ & 03 \\
\hline Total de espécimes: & & & & 78 \\
\hline
\end{tabular}

Legenda: sd $^{*}=$ sem data. APA = Área de Proteção Ambiental.

A partir deste levantamento foram documentados sete novos registros de espécies de Poales para o estado do Maranhão, sendo uma espécie de Cyperaceae (Bulbostylis consaguinea) e seis espécies de Poaceae (Bambusa vulgaris, Megathyrsus maximus, Paspalum eucomum, Paspalum guttatum, Paspalum lineare e Sacciolepis angustissima), ausentes na distribuição da lista da Flora do Brasil 2020 em Construção para o estado.

Muitas espécies de Poales depositadas no HABIT são resultados de coletas para produção de trabalhos de conclusão de curso por estudantes de Ciências Biológicas da UEMA, sediada em Caxias/MA. Assim, este município e em suas proximidades são os principais focos de coletas registrados no herbário. Além disso, o processo de identificação de grande parte do material botânico ocorreu com auxílio de taxonomistas especializados de outros estados do país, o que pode revelar evidência da escassez destes profissionais no estado em estudo.

Aproximadamente 99\% (212) dos espécimes de Cyperaceae Juss. presentes na coleção, foram coletados no Maranhão, nas Áreas de Proteção Ambiental Inhamum e Buriti do Meio, Parque Estadual do Mirador, margens de riachos, córregos e estradas dos municípios de Caxias, Balsas, Timon e povoados, com exceção de dois exemplares doados, $R$. consaguinea (Kunth) Boeckel proveniente de Brasília/DF, e Scleria bracteata Cav. proveniente do município de Patos/MG. Enquanto a famíla Poaceae Barn. apresentou um número maior de representantes doados, onde $79,49 \%$ (62) dos espécimes foram coletados no Maranhão, enquanto que $20,51 \%$ (16) dos espécimes foram doados por instituições provenientes dos estados de DF, MG, SP e TO (figura1 e 2). Essa permuta de materiais biológicos que ocorre entre acervos está relacionada a um dos objetivos de manter a comunicação científica entre os taxonomistas do mundo (JUDD et al., 2009), o que 
possibilita a identificação correta do material e o conhecimento da flora nacional e internacional.

Além disso, MONTEIRO \& SIANE (2009) destacam que a adoção desta prática de intercâmbio que vêm ocorrendo desde o século XVIII contribuiu para multiplicação dos acervos, e tem garantindo a sobrevivência de importantes registros após destruições causadas por incêndios, guerras, ou insetos.

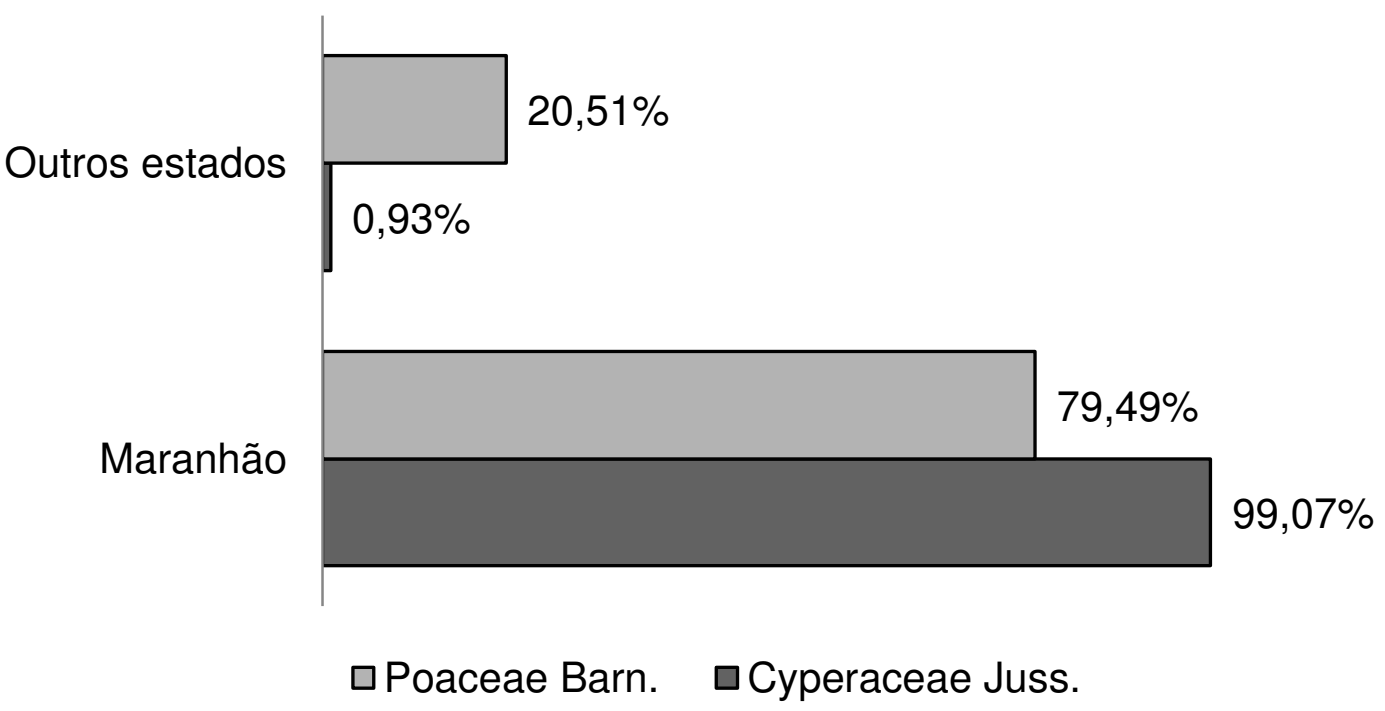

FIGURA 2. Percentual de espécimes de Cyperaceae Juss. e Poaceae Barn. depositadas no HABIT em relação ao local de origem.

De acordo com MARTINELLI \& MORAES (2013) 30 espécies de Cyperaceae, pertencentes a 12 gêneros, são representadas no catálogo do Livro Vermelho da Flora do Brasil com algum grau de ameaça, mas esse número pode elevar-se, uma vez que, quanto à distribuição geográfica, a família não está representada em sua real dimensão. Além disso, 67 espécies de Poaceae de 115 avaliadas entraram para a atual lista vermelha, sendo muitas endêmicas de campos rupestres, os quais tem sido impactados decorrente de ações antrópicas desordenadas.

Apenas duas espécies de Poales deste chekilist levantadas na coleção do HABIT apresentam-se caracterizadas quanto ao estado de conservação seguindo os critérios e categorias da IUCN, Rhynchospora tenuis e Raddiella esenbeckii, ambas exibindo fator menos preocupante de classificação (LC) (CNCFlora a; b, 2012). As demais espécies não estão avaliadas quanto a ameaça. 


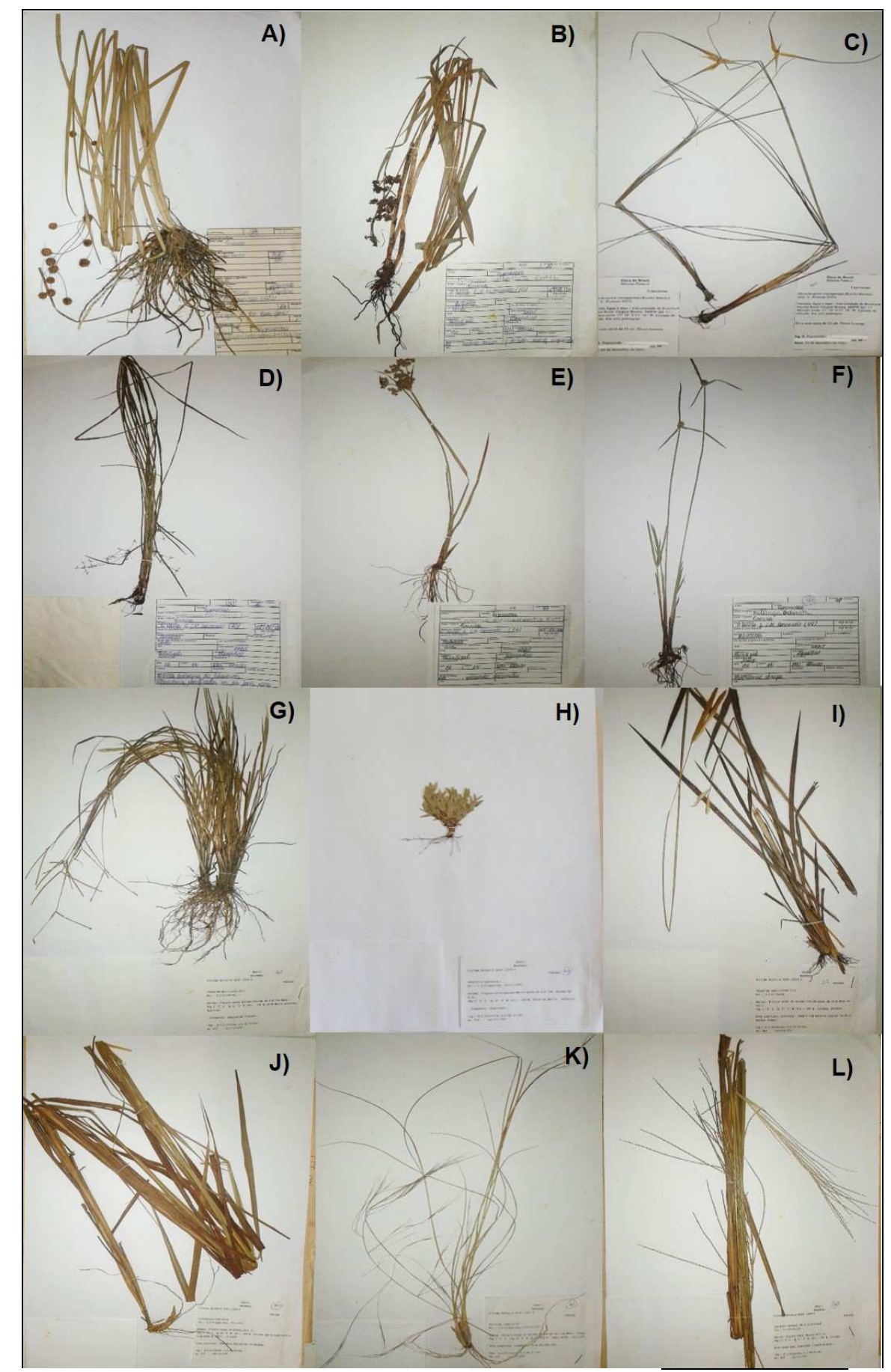

FIGURA 3. Espécies de Poales representadas no HABIT. Cyperaceae: A) Rhynchospora holoschoenoides, B) Fuirena umbellata, C) Rhynchospora consaguinea, D) Cyperus haspan, E) Cyperus surinamensis, F) Kyllinga odorata. Poaceae: G) Paspalum multicaule, H) Raddiella esenbeckii, I) Paspalum lanciflorum, J) Trachypogon espicatus, K) Aristida longifólia, L) Axonopus eminens.

\section{CONCLUSÃO}

Há baixa representatividade de Cyperaceae Juss. e Poaceae Barn. no Herbário Prof. Aluísio Bittencourt, entretanto, a maior parte das espécies depositadas nesta coleção é proveniente do Estado do Maranhão, fruto de pesquisas que ocorrem nessa região pela iniciativa dos próprios pesquisadores e estudantes de graduação vinculados à instituição onde se localiza o acervo. 
HABIT resguarda em suas instalações informações importantes, com potencial para contribuir em revelar a biodiversidade das famílias em questão representadas localmente, tais como a documentação de novas ocorrências, oferecendo assim, suporte para o desenvolvimento de estratégias de manejo e preservação em um bioma que há décadas apresenta-se em estado de alerta, como o Cerrado.

\section{REFERÊNCIAS}

ALISCIONI, S. S.; GIUSSANI, L. M.; ZULOAGA, F. O.; KELLOG, E. A. A molecular phylogeny of Panicum (Poaceae: Paniceae). Test of monophyly and phylogenetic placement with the Panicoideae. American Journal of Botany, v. 90, n. 5, p. 796821, 2003.

ALVES M.; ARAÚJO, A. C.; PRATA, A. P.; VITTA, F. A.; HEFleR, S. M.; TREVISAN, R. et al. Diversity of Cyperaceae in Brazil. Rodriguésia, v. 60, n. 4, p. 771-782, 2009. Disponível em: <http://repositorio.furg.br/handle/1/679>. Acesso em 03 de Março de 2017.

APG. III (Angiosperm Phylogeny Group). An update of the Angiosperm Phylogeny Group Classification for the orders and families of flowering plants: APG III. Botanical Journal of the Linnean Society, v. 161, n. 2, p. 105-121, 2009. Disponível em: <http://onlinelibrary.wiley.com/doi/10.1111/j.10958339.2009.00996.x/abstract>. doi: 10.1111/j.1095-8339.2009.00996.x

APG. IV (Angiosperm Phylogeny Group). An update of the Angiosperm Phylogeny Group Classification for the orders and families of flowering plants: APG IV. Botanical Journal of the Linnean Society, v. 181, p. 1-20, 2016. Disponível em: <http://reflora.jbrj.gov.br/downloads/2016_GROUP_Botanical\%20Journal\%20of\%20t he\%20Linnean\%20Society.pdf> doi: $10.1111 /$ boj. $1 \overline{2} 385$

BFG. Growing knowledge: an overview of Seed Plant diversity in Brazil. Rodriguésia, v. 66, n. 4, 2015.

BOLDRINI, I. I.; LONGHI-WAGNER, H. M. Poaceae no Rio Grande do Sul: diversidade, importância na fitofisionomia e conservação. Ciência Ambiental, n. 42, p. 71-92, 2011. Disponível em: <http://www.scielo.br/scielo.php?script=sci_nlinks\&ref=000075\&pid=S01023306201300010001700006\&lng=es>. Acesso em 10 de Março de 2017.

CNCFlora a. Rhynchospora tenuis in Lista Vermelha da flora brasileira versão 2012.2 Centro Nacional de Conservação da Flora. Disponível em <http://cncflora.jbrj.gov.br/portal/pt-br/profile/Rhynchospora tenuis>. Acesso em 26 abril 2017.

CNCFlora b. Raddiella esenbeckii in Lista Vermelha da flora brasileira versão 2012.2 Centro Nacional de Conservação da Flora. Disponível em <http://cncflora.jbrj.gov.br/portal/pt-br/profile/Raddiella esenbeckii>. Acesso em 26 abril 2017.

CONCEIÇÃO, G. M. Herbário Prof. Aluizio Bittencourt, Maranhão (HABIT). Bioscience, v. 4, n. 6, 2015. Disponível em: 
$<$ http://periodicos.unisanta.br/index.php/bio/article/view/477>. Acesso em 03 de Fevereiro de 2017.

FILGUEIRAS, T. S.; CANTO-DOROW, T. S.; CARVALHO, M. L. S.; DÓREA, M. C.; FERREIRA, F. M.; MOTA, A. C. et al. Poaceae. 2015. In: Lista de Espécies da Flora do Brasil. Jardim Botânico do Rio de Janeiro. Disponível em: $<$ http://floradobrasil.jbrj.gov.br/jabot/floradobrasil/FB193>. Acesso em 20 de Março de 2017.

Flora do Brasil 2020 em construção. Jardim Botânico do Rio de Janeiro. Disponível em: < http://floradobrasil.jbrj.gov.br/>. Acesso em: 04 Abril de 2017.

FREITAS, R. C. A.; SANTOS, M. L. G.; MATIAS, L. Q. Checklist das Monocotiledôneas do Ceará, Brasil. Caatinga, Mossoró, v. 24, n. 2, p. 75-84, 2011. Disponível em:

https://periodicos.ufersa.edu.br/revistas/index.php/sistema/article/view/1884>.

Acesso em: 20 de Março de 2017.

GOETGHEBEUR, P. Cyperaceae. In: HUBER, K. H.; RUDALL, P. J.; STEVENS, P. S.; STÜTZEL, T. The Families and Genera of Vascular Plants. IV: Flowering plants - monocotyledons. Berlin: Springer-Verlang, p. 141-190, 1998.

GOVAERTS, R.; SIMPSON, D. A.; GOETGHEBEUR, P.; WILSON, K. L.; EGOROVA, T.; BRUHL, J. World checklist of Cyperaceae. Kew: The Board of Trustees of the Royal Botanical Garden, 2007.

GPWG - The Grass Phylogeny Working Group. 2001. Phylogeny and subfamilial classification of the grasses (Poaceae). Annals of the Missouri Botanical Garden v. 88, n. 3, p. 373-457, 2013.

JUDD, W. S.; CAMPBELL, C. S.; KELLOGG, E. A.; STEVENS, P. F.; DONOGHUE, M. J. Sistemática vegetal: um enfoque filogenético. 3. ed. Porto Alegre: Artmed, $p$. 612, 2009.

KEARNS, D. M.; THOMAS, W. W. TUCKER, G.; KRAL, R.; CAMELBEKE, K.; SIMPSON, D. A. et al. Cyperaceae. In: BERRY, P.E.; YATSKIEVYCH, K.; HOLST, B. K. Flora of the Venezuelan Guayana, Missouri Botanical Garden, Press, St. Louis, n. 4, p. 486-663, 1998.

LUCEÑO, M.; ALVES, M. Clave de los géneros de ciperáceas de Brasil y novedades taxonómicas y corologicas en la família. Candollea, v. 52, p.185-191, 1997.

MARINHO-FILHO, J.; MACHADO, R. B.; HENRIQUES, R. P. B. Evolução do conhecimento e da conservação do Cerrado brasileiro. P. 15-34, 2010. In: DINIZ, I. R.; MARINHO-FILHO, J.; MACHADO, R. B.; CAVALCANTI, R. B. (Org.). Cerrado: conhecimento científico quantitativo como subsídio para ações de conservação. Brasília: Thesaurus, p.13-31, 2010. Disponível em: file:///C:/Users/paula\%20regina/Downloads/cerrado_introducao.pdf> Acesso em: 03 de Abril de 2017. 
MARTINELLI, G.; MORAES, M. A. Livro vermelho da flora do Brasil. 1. ed. Rio de Janeiro: Instituto de Pesquisas Jardim Botânico do Rio de Janeiro, p. 1100, 2013. Disponível em: <cncflora.jbrj.gov.br/arquivos/arquivos/pdfs/LivroVermelho.pdf>. Acesso em 25 de Março de 2017.

MENDES, K.; GOMES, P.; ALVES, M. Floristic inventory of a zone of ecological tension in the Atlantic Forest of Northeastern Brazil. Rodriguésia, v. 61, n. 4, p. 669676, 2010. Disponível em: <http://rodriguesiaseer.jbrj.gov.br/index.php/rodriguesia/article/view/182/24>. Acesso em: 01 Abril de 2017.

MITTERMEIER, R. A.; MYERS, N.; GIL, P. R.; MITTERMEIER, C. G. Hotspost. Earth's biologically richest and most endangered terrestrial ecoregions. CEMEX, Conservation International, p. 431, 2004.

MONTEIRO, S. S.; SIANI, A. C. A Conservação de Exsicatas em Herbários: Contribuição ao Manejo e Preservação. Fitos, v. 4, n. 2, p. 14, 2009. Disponível em: <http://revistafitos.far.fiocruz.br/index.php/revista-fitos/article/view/95/94>. Acesso em: 04 Março de 2017.

NUNES, C. S.; CONCEIÇAO, G. M.; SILVA, E. O. Levantamento das espécies de Cyperaceae juss. na área de Proteção Ambiental Municipal do Inhamum, Caxias, Maranhão, Brasil. Enciclopédia Biosfera, v. 8, n.15; p. 1796, 2012. Disponível em: <http://www.conhecer.org.br/enciclop/2012b/ciencias\%20biologicas/especies.pdf>. Acesso em: 04 Abril de 2017.

OLIVEIRA, R. C.; VALLS, J. F. M. Paspalum L. 2001. In: WANDERLEY, M. G. L et al. (Eds), Flora fanerogâmica do Estado de São Paulo, Poaceae, HUCITEC, São Paulo, Brasil, v. 1, p. 191-227.2001.

OSBORNE, C. P.; VISSER, V.; CHAPMAN, S.; BARKER, A.; FRECKLETON, R. P.; SALAMIN, N. et al. GrassPortal: an online ecological and evolutionary data facility for the grasses. South African Journal of Botany, v.77, n.2, p. 566-566, 2011.

PEIXOTO, A. L.; MORIM, M. P. Coleções botânicas: documentação da biodiversidade brasileira. Ciência \& Cultura, v. 55, n. 3, p. 21-48, 2003. Disponível em: <http://cienciaecultura.bvs.br/pdf/cic/v55n3/a16v55n3.pdf>. Acesso em: 20 e Março de 2017.

PRATA, A. P.; VITTA, F.; ALVES, M. HEFLER, S.; BORGES, R. A. X.; HERING, R. L. O. et al. Cyperaceae. In: MARTINELLI, G.; MORAES, M. A. Livro vermelho da flora do Brasil. 1. ed. Rio de Janeiro: Instituto de Pesquisas Jardim Botânico do Rio de Janeiro, p. 468-473, 2013.

QUEIRÓS, S. G.; CONCEIÇÃO, G. M.; NASCIMENTO, J. M. Checklist das espécies de Angiospermas doadas sob acervo, na coleção Botânica do Laboratório de Biologia Vegetal, da Universidade Estadual do Maranhão. Agrarian Academy, Centro Científico Conhecer, Goiânia, v. 1, n. 1, p. 27, 2014. Disponível em: <http://www.conhecer.org.br/Agrarian\%20Academy/2014a/CHEKLIST.pdf>. Acesso em: 25 de Fevereiro de 2017. 
REZENDE, A. V.; WALTER, B. M. T.; FAGG, C. W.; FELFILI, J. M.; SILVA-JÚNIOR, M. C.; NOGUEIRA, P. E. Flora vascular do 15 bioma cerrado. In: SANO, S. M.; ALMEIDA, S. P.; RIBEIRO, J. F. Cerrado: Ecologia e flora. Embrapa, Brasília. p. 1028-1059, 2008. Disponível em: <http://livraria.sct.embrapa.br/liv_resumos/pdf/00082110.pdf>. Acesso em: 02 de Abril de 2017.

RIBEIRO, A. R. O.; PRATA, A. P. N.; CAMACHO, R. G. V.; OlIVEIRA, O. F.; OLIVEIRA, R. C. Cyperaceae do rio Apodi-Mossoró, Estado do Rio Grande do Norte, Brasil. Hoehnea, v. 41, n. 2, p. 149-171, 2014. Disponível em: <http://www.scielo.br/scielo.php?script=sci_arttext\&pid=S2236-

89062014000200001> doi: 10.1590/S2236-89062014000200001

RUA, G. H. Estudos filogenéticos em Paniceae: os casos de Paspalum e Digitaria. In: MARIATH, J. E. A.; SANTOS, R. P. Os avanços da botânica no início do século XXI: morfologia, fisiologia, taxonomia, ecologia e genética. Porto Alegre: Sociedade Botânica do Brasil. cap. 35, p.170-173, 2006.

SANTOS, D. S.; CONCEIÇAO, G. M.; SILVA, E. O. Contribuição ao conhecimento das espécies de Cyperaceae da área de Proteção Ambiental Municipal do Inhamum, Caxias, Maranhão. Agrarian Academy, Centro científico conhecer, Goiânia, v.1, n.1; p. 312, 2014. 20 Disponível <http://www.conhecer.org.br/Agrarian\%20Academy/2014a/contribuicao.pdf>. Acesso em: 26 de Março de 2017.

STRONG, M. T. Taxonomy and Distribution of Rhynchospora (Cyperaceae) In the Guianas, South America. Contributions from the United States National Herbarium, n. 53, p. 225, 2006.

TREVISAN, R.; BOLDRINI, I. I. Novelties in Eleocharis Ser. Tenuissimae (Cyperaceae) and a Key to the Species of the Series Occurring In Brazil. Systematic Botany, n. 35, p. 504-511, 2010.

VIEIRA, A. O. S.; GASPER A. L. Herbários do Brasil. Bioscience, v. 4, n. 6, 2015. Disponível em: <http://periodicos.unisanta.br/index.php/bio/article/view/447>. Acesso em: 07 de Março de 2017. 